PHYSICAL REVIEW D 87, 041501(R) (2013)

\title{
Self-force as a cosmic censor
}

\author{
Peter Zimmerman, Ian Vega, and Eric Poisson \\ Department of Physics, University of Guelph, Guelph, Ontario N1G 2W1, Canada
}

Roland Haas

TAPIR, Mailcode 350-17, California Institute of Technology, Pasadena, California 91125, USA

(Received 16 November 2012; published 8 February 2013)

\begin{abstract}
We examine Hubeny's scenario according to which a near-extremal Reissner-Nordström black hole can absorb a charged particle and be driven toward an over-extremal state in which the charge exceeds the mass, signaling the destruction of the black hole. Our analysis incorporates the particle's electromagnetic self-force and the energy radiated to infinity in the form of electromagnetic waves. With these essential ingredients, our sampling of the parameter space reveals no instances of an overcharged final state, and we conjecture that the self-force acts as a cosmic censor, preventing the destruction of a nearextremal black hole by the absorption of a charged particle. We argue, on the basis of the third law of black hole mechanics, that this conclusion is robust and should apply to attempts to overspin a Kerr black hole.
\end{abstract}

DOI: 10.1103/PhysRevD.87.041501

PACS numbers: 04.70.Bw, 04.20.Dw

\section{INTRODUCTION}

The expectation that curvature singularities arising from the gravitational collapse of matter in general relativity should be shielded from view by event horizons was codified in a cosmic censorship conjecture, first formulated by Penrose in 1969 [1]. While a proof is still lacking, the conjecture is supported by numerous examples [2], but it is also challenged by a number of potential counterexamples. Some of these involve matter models that would be dismissed as insufficiently physical (such as a pressureless fluid), but some are sufficiently serious to warrant a close examination. Among these is the critical collapse of fundamental matter fields, as investigated by Choptuik and his collaborators [3,4]; in such cases the critical solution describes a naked singularity, but its realization requires an initial configuration that is infinitely finely tuned. Another is the endpoint of the Gregory-Laflamme instability of a five-dimensional black string [5,6], which features a horizon breaking up into ever smaller beads joined by ever thinner filaments, leading to the formation of a naked singularity; but there are no four-dimensional analogues to this instability.

Another avenue for violating cosmic censorship was identified by Hubeny [7], who noticed that a near-extremal Reissner-Nordström black hole, possessing a charge $Q$ that is almost as large as its mass $M$ (in geometrized units in which $G=c=1$ ), can absorb a test particle of such charge $q$, mass $m$, and energy $E_{0}$ that the final configuration cannot be a black hole: the final charge $Q+q$ exceeds the final mass $M+E_{0}$, signaling the presumed destruction of the event horizon. As we shall review in Sec. III, Hubeny identified an open region of parameter space that corresponds to such overcharging scenarios, revealing that they constitute a plausible threat to cosmic censorship.
This theme was further explored by Hod [8] and Jacobson and Sotiriou [9], who found that a near-extremal Kerr black hole can absorb a test particle and be driven toward a final state with too much angular momentum to be a black hole. In these scenarios it is important that the initial black hole be in a near-extremal state; an already extremal black hole would necessarily repel the particle and prevent it from crossing the event horizon [10]. And as we shall explain in Sec. V, it is also important that the particle be a point particle with a vanishing physical size [11]: the process must be discontinuous. We therefore exclude from our considerations attempts to overcharge or overspin a black hole by continuous processes-for example, by using waves instead of particles $[12,13]$ such attempts will necessarily fail.

Another important aspect of the overcharging and overspinning scenarios is that they were analyzed on the basis of an approximate description of the relevant physics. First, the absorbed particle was modeled as a test particle, and all self-force, self-energy, and radiative effects were ignored. Second, the gravitational influence of the particle on the black hole spacetime was not taken into account; while one could show that the final configuration had too much charge or angular momentum to be a black hole, the analysis could not describe how the black hole gets dynamically destroyed. In this paper we address the first limitation; the second limitation will not be addressed, but our analysis indicates that the black hole will not be destroyed by the absorption of a particle.

Other researchers have attempted to incorporate the important influence of the particle's self-force, and of the radiation emitted during the absorption, on the overcharging and overspinning scenarios. Hubeny, in her original work on the subject [7], recognized the limitations of the test particle analysis and attempted to incorporate 
self-force effects through a local approximation, to be described in Sec. III below. Isoyama, Sago, and Tanaka investigated self-energy effects [14], suggesting that the existence of a turning point in the particle's motion is compatible with a final state that is not overcharged. Barausse, Cardoso, and Khanna incorporated the gravitational radiation emitted by a particle attempting to overspin a Kerr black hole $[15,16]$. These attempts were incomplete: Hubeny's self-force was approximate, Isoyama et al. could not state that the self-force necessarily produces a turning point when the black hole is about to become overcharged, and Barausse et al. could not account for conservative self-force effects.

Our purpose in this paper is to provide a fuller analysis of the overcharging scenarios. While our attempt is still partial (as we shall explain in Sec. II), it is much more complete than the ones reviewed previously. And while these attempts could not rule out all cases of overcharged final states, we provide evidence that when both conservative and dissipative aspects of the self-force are taken into account, there are no overcharged final states. We therefore present a case that the electromagnetic self-force acts as a cosmic censor, preventing the destruction of a nearextremal black hole by the absorption of a charged particle.

Our analysis benefits from the technical developments of the last decade, reviewed in Ref. [17], which permit the routine computation of self-forces in curved spacetime. These computations are relatively straightforward when the background spacetime is spherically symmetric, and this motivates us to limit the scope of our work to the overcharging scenarios. An analysis of the overspinning scenarios would require the computation of the gravitational self-force on a particle plunging with high angular momentum toward a rapidly rotating Kerr black hole, and this is beyond the current state of the art. There are also no techniques currently available to calculate the gravitational self-force on a spinning particle.

\section{ELECTROMAGNETIC SELF-FORCE AND RADIATED ENERGY}

To calculate the self-force acting on a charged particle falling toward a charged black hole is a formidable undertaking that is made extremely difficult by the coupling of electromagnetic and gravitational perturbations in the background Reissner-Nordström spacetime. The metric is a solution to the Einstein field equations with an energymomentum tensor $T$ that is quadratic in the electromagnetic field tensor $F$. The introduction of a charged particle creates a perturbation $\delta F_{1}$ to the field tensor, and a perturbation $\delta T \sim F \delta F_{1}$ to the energy-momentum tensor; this produces a metric perturbation $\delta g_{2}$ that must be added to the direct perturbation $\delta g_{1}$ created by the particle's mass. Furthermore, the metric perturbation creates a disturbance $\delta F_{2}$ in the background field tensor, which must be added to $\delta F_{1}$. The perturbative problem is inherently coupled, and techniques to calculate self-forces in such circumstances are not yet available. Our treatment will therefore be incomplete, in that we shall eliminate the gravitational sector from the perturbative problem; our electromagnetic perturbation lives in a fixed background spacetime, and no attempt will be made to couple it to gravity. A partial justification can be given: When the particle's chargeto-mass ratio is very large, the gravitational self-force associated with $\delta g_{1}$ can be neglected in front of the electromagnetic self-force associated with $\delta F_{1}$, but the neglect of $\delta g_{2}$ and $\delta F_{2}$ cannot be so easily justified. We shall proceed nevertheless, and argue in Sec. V that our treatment captures the essential aspects of the self-force.

We consider a point particle falling radially toward a Reissner-Nordström black hole. The spacetime metric is

$$
d s^{2}=-f d t^{2}+f^{-1} d r^{2}+r^{2} d \Omega^{2},
$$

in which $f=1-2 M / r+Q^{2} / r^{2}$ and $d \Omega^{2}=d \theta^{2}+$ $\sin ^{2} \theta d \phi^{2}$. The background electromagnetic field tensor has $F_{r t}=Q / r^{2}$ as its only nonvanishing component, and the particle creates a perturbation $\delta F_{r t}$ that we decompose as

$$
\delta F_{r t}=\frac{1}{r^{2}} \sum_{\ell m} \Phi_{\ell m}(t, r) Y_{\ell m}(\theta, \phi)
$$

in terms of spherical harmonics; the other components of the perturbation play no role in our analysis. Each mode $\Phi_{\ell_{m}}$ of the perturbation satisfies the partial differential equation

$$
-\partial_{t t} \Phi+f \partial_{r}\left(f \partial_{r} \Phi\right)-\frac{\ell(\ell+1)}{r^{2}} f \Phi=S,
$$

in which

$$
S=4 \pi r^{2} f\left(\partial_{t} j_{r}-\partial_{r} j_{t}-2 j_{t} / r\right)
$$

is a source term constructed from

$$
\begin{aligned}
& j_{t}=-q \sqrt{\frac{2 \ell+1}{4 \pi}} \frac{F}{R^{2}} \delta(r-R), \\
& j_{r}=q \sqrt{\frac{2 \ell+1}{4 \pi}} \frac{\dot{R}}{F R^{2}} \delta(r-R),
\end{aligned}
$$

the modes of the current density $j_{\alpha}$, with $r=R(t)$ describing the world line, $\dot{R}=d R / d t$, and $F=f(r=R)$. The world line is obtained by integrating the differential equation

$$
\frac{d R}{d t}=-\frac{F}{E_{0}-q Q / R} \sqrt{\left(E_{0}-q Q / R\right)^{2}-m^{2} F} .
$$

Equation (3) is integrated numerically, making use of a finite difference method devised by Lousto and Price [18]; the method is designed to provide an exact treatment of the delta functions on the right-hand side of Eq. (3). 
The integration of Eq. (3) requires the specification of $\Phi(t, r)$ and $\partial_{t} \Phi(t, r)$ at $t=0$. These initial conditions are unknown, and in most of the self-force literature $[19,20]$ it has proved adequate to adopt the trivial configuration $\Phi(0, r)=\partial_{t} \Phi(0, r)=0$, in spite of the obvious violation of the constraint equations at $t=0$. In the usual context in which the particle moves slowly and can be followed for a very long time, the unphysical burst of radiation that accompanies the sudden creation of a particle at $t=0$ is of no concern; the radiation travels away from the particle at the speed of light and leaves the numerical domain before the self-force is evaluated. The present context is less forgiving. As we shall see, our particles move extremely fast at $t=0$, and this gives little opportunity for the radiation to peel away from the particle; and since the particle takes little time to reach the black hole, the numerical noise is still present when we evaluate the selfforce. This unfavorable circumstance represents a significant obstacle against high-precision computations of the self-force. In practice we were able to mitigate this difficulty by starting the integration when the particle is extremely far away from the black hole, and restarting it after some time on a smaller numerical grid, using the previously generated results as initial data for the new run. But while this technique does a good job at eliminating most of the noise, some remains, and it continues to limit the accuracy of our computations for high-speed particles.

The electromagnetic self-force acting on the charged particle is computed according to

$$
f^{r}=\frac{q}{m}\left(E_{0}-\frac{q Q}{R}\right) \delta F_{r t}^{\mathrm{R}},
$$

in which $f^{r}$ is the radial component of the force and $\delta F_{r t}^{\mathrm{R}}$ is the regularized electromagnetic field produced by the particle; this differs from the retarded solution $\delta F_{r t}$ to Maxwell's equations by the Detweiler-Whiting singular field [21], which diverges at the particle's position but is known not to contribute to the self-force. In practice the regularized field is computed by implementing a modesum regularization [22] according to which

$$
\delta F_{r t}^{\mathrm{R}}=\sum_{\ell}\left[\left(\delta F_{r t}\right)_{\ell}-\left(\ell+\frac{1}{2}\right) A-B+\cdots\right],
$$

where $\left(\delta F_{r t}\right)_{\ell}=r^{-2} \sum_{m} \Phi_{\ell m} Y_{\ell m}$ evaluated at the particle's position, and

$$
A=\frac{q}{R^{2}}, \quad B=\frac{q E_{0}}{2 m R^{2}}-\frac{q^{2} Q}{m R^{3}}
$$

are regularization parameters calculated by adapting the recipe described in Sec. X of Ref. [23] to the radial trajectories considered here. The remaining terms in the mode sum are given by such expressions as $\left[\left(\ell-\frac{1}{2}\right) \times\right.$ $\left.\left(\ell+\frac{3}{2}\right)\right]^{-1}$ multiplied by additional regularization parameters; these sum to zero, and keeping these terms accelerates the convergence of the mode sum when it is necessarily truncated to a maximum value $\ell_{\max }$. The regularized mode sum provides a powerful diagnostic of numerical accuracy; the computations are deemed to be unreliable when $\left(\delta F_{r t}^{\mathrm{R}}\right)_{\ell}$ fails to fall off as $\ell^{-2}$ for large $\ell$ after subtraction of the $A$ and $B$ terms.

The influence of the self-force on the particle's motion can be incorporated by promoting $E_{0}$ to a dynamical variable $E(R)$ in Eq. (6), which is related to the self-force by $d E / d R=\delta F_{r t}^{\mathrm{R}}$. A central question is whether the selfforce succeeds in repelling the particle before it reaches the event horizon; this will be the case when $E$ decreases to the extent that it becomes equal to $q Q / R+m \sqrt{F}$, which signals the presence of a turning point. This occurs when $\delta F_{r t}^{\mathrm{R}}>0$ and the self-force is repulsive.

The energy radiated in the form of electromagnetic waves by the infalling particle can also be obtained on the basis of the mode functions $\Phi_{\ell m}$ evaluated in the limit $r \rightarrow \infty$. The rate at which energy is radiated to infinity is given by

$$
\frac{d E_{\infty}}{d t}=\sum_{\ell m} \frac{\left|\partial_{t} \Phi_{\ell m}\right|^{2}}{\ell(\ell+1)}
$$

This can be integrated with respect to $t$ to obtain $E_{\infty}$, the total energy radiated.

\section{MONTE CARLO SAMPLING OF OVERCHARGING TRAJECTORIES}

The self-force and the radiated energy can be computed once a choice of trajectory $R(t)$ is made. To guide this choice, we recall Hubeny's test particle analysis, which involves a black hole in a near-extremal state with $Q / M=$ $1-2 \epsilon^{2}$, where $\epsilon$ is small and positive. The particle's charge, energy, and mass are parametrized as

$$
q / M=a \epsilon, \quad E_{0} / M=a \epsilon-2 b \epsilon^{2}, \quad m / M=c \epsilon,
$$

in which $(a, b, c)$ are dimensionless and of order unity. Hubeny showed that particles with $a>1,1<b<a$, and $c<\sqrt{a^{2}-b^{2}}$ produce a final configuration with $Q+q>$ $M+E_{0}$, which is overcharged relative to a black hole state. This analysis ignores self-force effects, and it ignores the energy radiated by the infalling particle. It can be shown that incorporation of these effects does not affect the bound on $a$ or the upper bound on $b$. The lower bound on $b$, however, is affected, because the overcharging condition becomes

$$
Q+q>M+E_{0}-E_{\infty}
$$

to account for the energy radiated to infinity; since $q=O(\epsilon), E_{\infty} / M$ can be parametrized as $\alpha \epsilon^{2}$ for some dimensionless quantity $\alpha$, and $b$ 's lower bound becomes $b>1-\frac{1}{2} \alpha$. While radiative effects alter the overcharging condition, the self-force determines whether a turning point will be encountered before the particle reaches the 
event horizon. To ensure that the particle's charge-to-mass ratio is large (so that we can ignore the gravitational selfforce), we require that $c \ll a$.

It would be hopeless to perform self-force computations for the infinite number of trajectories that can potentially lead to overcharging scenarios; indeed, each self-force computation requires several hours of CPU time on a standard workstation, and each computation must be carefully examined to ensure that it is not vulnerable to initial data noise. To work around these prohibitive costs, we first performed a Monte Carlo search of the parameter space by implementing crude approximations for the self-force and the energy radiated. For the purposes of this search, we approximated the full self-force by a local approximation $[24,25]$ given by

$$
f_{\text {local }}^{\alpha}=\frac{1}{3} q^{2}\left(g^{\alpha \beta}+u^{\alpha} u^{\beta}\right)\left(2 \frac{D a_{\beta}}{d \tau}+R_{\beta \gamma} u^{\gamma}\right),
$$

in which $D a_{\beta} / d \tau$ is the particle's acceleration covariantly differentiated with respect to proper time, and $R_{\beta \gamma}$ is the spacetime's Ricci tensor. The flux of radiated energy is approximated by a relativistic version of Larmor's formula, $d E_{\infty} / d t \simeq \frac{2}{3} q^{2} a^{\alpha} a_{\alpha}$, which is integrated to yield $E_{\infty}$. With these crude ingredients, our Monte Carlo search involved 10000 trajectories sampled uniformly in the $(a, b, c)$ parametrization; the samples were taken within the intervals $1<a<100,-200<b<a$, and $0<c<a$, and for all samples we set $\epsilon=0.01$. Of these, only 27 trajectories fulfilled the requirements for an overcharging scenario: they managed to penetrate the black hole in spite of the repulsive action of the self-force, and they satisfied the condition of Eq. (12). The remaining cases were dismissed either because the local self-force produces a turning point before the particle reaches the event horizon, or because the Larmor formula indicates that the overcharging condition is not satisfied.

\section{ACCURATE COMPUTATIONS}

The Monte Carlo search was followed up with accurate computations of the self-force and radiated energy for a

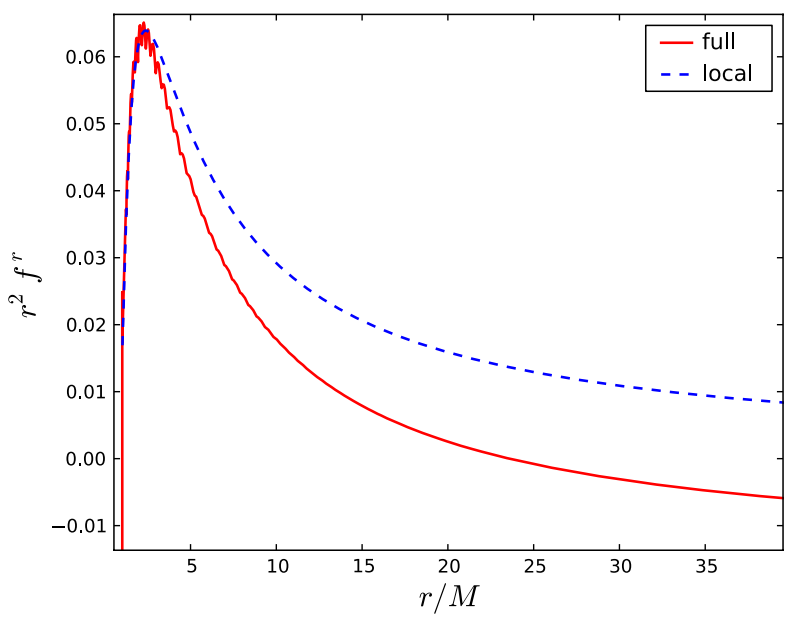

FIG. 1 (color online). Plot of $r^{2} f^{r}$ as a function of $r / M$ for the first case listed in Table I. The dashed line (blue) is the local approximation. The solid line (red) is the result of our computation. The local approximation overestimates the self-force except when the particle is very close to the event horizon, where its action is most important. The oscillations seen below $r / M=5$ are a manifestation of numerical noise associated with an unphysical burst of radiation taking place at $t=0$.

much smaller sample of trajectories. In general, we found that close to the black hole, the actual self-force is well approximated by the local expression; the level of discrepancy never exceeds $10 \%$. We also find that the radiated energy is rather crudely approximated by the Larmor formula, at a typical level of $40 \%$ accuracy.

Choosing among the cases that do not produce an overcharged final state because of the existence of a turning point, we find that the actual self-force tends to be larger than the local approximation, confirming the failure of the particle to cross the event horizon. Choosing among the cases for which the particle crosses the horizon but Eq. (12) is not satisfied, we find that the actual self-force also fails to produce a turning point, but the accurate computation of $E_{\infty}$ confirms that the final state is not overcharged.

TABLE I. Three sampled trajectories that were declared to produce an overcharged final state in the Monte Carlo search. The table lists the values of $(a, b, c)$ that parametrize the choice of trajectory. It also specifies the initial state of the particle at $t=0$; to obtain reliable results for the self-force and radiated energy, we must begin the integrations at a very large radius, and with initial speeds that approach the speed of light. The table indicates whether the actual self-force allows the particle to cross the event horizon; in all cases, the answer is positive. Finally, the table indicates whether the final state satisfies the overcharging condition of Eq. (12); while all answers would have been positive under the Larmor approximation, they are actually negative when the radiated energy is computed accurately.

\begin{tabular}{lcccccc}
\hline \hline$a$ & $b$ & $c$ & $R / M$ & $\dot{R}$ & Crossing? & Overcharging? \\
\hline 3.728 & -46.161 & 0.7535 & 12,000 & 0.987 & Yes & No \\
3.825 & -125.73 & 0.7829 & 20,000 & 0.992 & Yes & No \\
3.910 & -146.10 & 0.7120 & 30,000 & 0.994 & Yes & No \\
\hline \hline
\end{tabular}


Finally, choosing among the cases that did produce an overcharged final state (see Table I) reveals that the actual self-force is smaller than the local approximation (see Fig. 1), so that it cannot succeed in producing a turning point. For these cases, however, we find that the Larmor formula overestimates the radiated energy, producing a final mass $M_{\text {final }}=M+E_{0}-E_{\infty}$ that is smaller than the actual value; so while $M_{\text {final }}$ was declared to be smaller than $Q_{\text {final }}=Q+q$ under the Larmor approximation, we actually have $M_{\text {final }}>Q_{\text {final }}$ and a final state that is not overcharged.

\section{THIRD LAW OF BLACK HOLE MECHANICS}

Our limited sample of the parameter space has revealed no instances of an overcharged final state. The electromagnetic self-force seems to act as a cosmic censor, preventing the destruction of a near-extremal black hole by the absorption of a charged particle. To reinforce this conclusion, we elaborate an argument that suggests that it is robust. In particular, we believe that the conclusion is not limited by our incomplete sampling of the parameter space and our neglect of the gravitational coupling. We believe that it would hold just as well in attempts to overspin a Kerr black hole. The argument is based on the third law of black hole mechanics.

As formulated and proved by Israel [26], the third law states that "a nonextreme black hole cannot become extremal (i.e., lose its trapped surfaces) at a finite advanced time in any continuous process in which the stress-energy tensor of accreted matter stays bounded and satisfies the weak energy condition in a neighborhood of the outer apparent horizon." (The mathematics behind the theorem were further developed by Andersson and his collaborators [27]). An important aspect of Israel's theorem is that it is formulated in terms of the black hole's apparent horizon and does not rely on the global existence of an event horizon (which would be akin to assuming the validity of cosmic censorship). Another important aspect is that the third law requires a continuous process involving a bounded stress-energy tensor, and therefore it does not apply to the point-particle scenarios considered here. The third law, however, implies that any attempt to overcharge or overspin a black hole based on continuous processes will necessarily fail.

Our interest here is in a discontinuous process associated with a point particle. Let us, however, consider a small but extended body that is threatening to overcharge or overspin a nearly extremal black hole. This body satisfies the conditions of the theorem, and it cannot succeed in destroying the black hole; it cannot even succeed in bringing the black hole to extremality. What is the mechanism behind this failure?

For the region of parameter space examined here, there would be no obstacle against overcharging or overspinning the black hole if the body were modeled as a test body in the black hole's background spacetime. The mechanism must therefore be provided by backreaction effects. For these overcharging or overspinning cases, therefore, backreaction effects must force the body to turn around before the event horizon is reached. In other words, the net selfforce acting on the extended body must provide the required mechanism that prevents the body from violating the third law. The point particle evades the third law, but it is clear on physical grounds that in a regime in which the extended body is sufficiently small, the self-force acting on a point particle will be indistinguishable from the self-force acting on the extended body. And if the self-force manages to prevent the extended body from destroying the black hole, it must do the same for the point particle. Our conclusion, therefore, is that the self-force acts as a cosmic censor under all such circumstances.

While this argument appears to us to be most plausible, we acknowledge that it does not amount to a proof that a point particle can never be exploited to overcharge or overspin a black hole. For example, it is conceivable that an extended body threatening to overcharge or overspin would break apart before reaching the horizon, with its largest fraction turning around and a suitably small fraction being absorbed by the black hole, keeping it in a nonextremal state. If such a circumstance were to arise, it would be difficult to argue that the self-force on the point particle would be indistinguisable from the self-force on the extended body. We would, however, dismiss this scenario as extremely unlikely when the body is sufficiently small. Indeed, the destruction of the body would require strong tidal forces (produced either by the black hole or the body's self-force), and these will necessarily scale linearly with the body's size; while a large body might indeed be broken up by tidal forces, a small body will not.

\section{CONCLUSION}

We have presented two lines of argument against the destruction of a near-extremal black hole by the absorption of a charged particle. The first relies on a calculation of the electromagnetic self-force and energy radiated that neglects the gravitational sector of the perturbation. The second relies on the third law of black hole mechanics and the expectation that the motion of a point particle cannot be distinguished from the motion of a suitably small body. Each line of argument is incomplete. But we believe that taken together, they amount to a solid case in favor of the conjecture that the self-force can act as a cosmic censor.

\section{ACKNOWLEDGMENTS}

We acknowledge numerous discussions with colleagues, including Leor Barack, Luis Lehner, Adam Pound, and Bob Wald. This work was supported by the Natural Sciences and Engineering Research Council of Canada. 
[1] R. Penrose, Riv. Nuovo Cimento 1, 252 (1969).

[2] R. M. Wald, arXiv:gr-qc/9710068.

[3] M.W. Choptuik, Phys. Rev. Lett. 70, 9 (1993).

[4] C. Gundlach and J.M. Martin-Garcia, Living Rev. Relativity 10, 5 (2007).

[5] R. Gregory and R. Laflamme, Phys. Rev. Lett. 70, 2837 (1993).

[6] L. Lehner and F. Pretorius, Phys. Rev. Lett. 105, 101102 (2010).

[7] V.E. Hubeny, Phys. Rev. D 59, 064013 (1999).

[8] S. Hod, Phys. Rev. D 66, 024016 (2002).

[9] T. Jacobson and T.P. Sotiriou, Phys. Rev. Lett. 103, 141101 (2009); 103, 209903(E) (2009).

[10] R. M. Wald, Ann. Phys. (N.Y.) 82, 548 (1974).

[11] S. Hod and T. Piran, Gen. Relativ. Gravit. 32, 2333 (2000).

[12] S. Hod, Phys. Rev. Lett. 100, 121101 (2008).

[13] J. Kommemi, arXiv:1107.0949.

[14] S. Isoyama, N. Sago, and T. Tanaka, Phys. Rev. D 84, 124024 (2011).

[15] E. Barausse, V. Cardoso, and G. Khanna, Phys. Rev. Lett. 105, 261102 (2010).
[16] E. Barausse, V. Cardoso, and G. Khanna, Phys. Rev. D 84, 104006 (2011).

[17] E. Poisson, A. Pound, and I. Vega, Living Rev. Relativity 14, 7 (2011).

[18] C. O. Lousto and R.H. Price, Phys. Rev. D 55, 2124 (1997).

[19] R. Haas, Phys. Rev. D 75, 124011 (2007).

[20] L. Barack and N. Sago, Phys. Rev. D 81, 084021 (2010).

[21] S. Detweiler and B.F. Whiting, Phys. Rev. D 67, 024025 (2003).

[22] L. Barack and A. Ori, Phys. Rev. D 66, 084022 (2002).

[23] M. Casals, E. Poisson, and I. Vega, Phys. Rev. D 86, 064033 (2012).

[24] B. S. DeWitt and R. W. Brehme, Ann. Phys. (N.Y.) 9, 220 (1960).

[25] J. M. Hobbs, Ann. Phys. (N.Y.) 47, 141 (1968).

[26] W. Israel, Phys. Rev. Lett. 57, 397 (1986).

[27] L. Andersson, M. Mars, J. Metzger, and W. Simon, Classical Quantum Gravity 26, 085018 (2009). 